\title{
The association between mental rotation capacity and motor impairment in children with obesity - an exploratory study
}

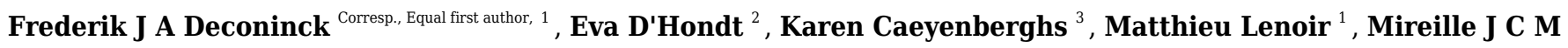
Augustijn ${ }^{\text {Equal first author, } 1}$

1 Department of Movement and Sports Sciences, Ghent University, Ghent, Belgium

2 Faculty of Physical Education and Physiotherapy, Vrije Universiteit Brussels, Brussels, Belgium

3 Mary MacKillop Institute for Health Research, Australian Catholic University, Melbourne, Victoria, Australia

Corresponding Author: Frederik J A Deconinck

Email address: frederik.deconinck@ugent.be

Background. Motor impairments are relatively common in children with obesity and evidence suggests that these difficulties go beyond those expected based on the extra weight. This study aimed to investigate the mental rotation capacity in children with obesity, i.e. the ability to mentally view and rotate spatial information, which is a function of both visual-spatial and action representation processes. More in particular, we examined whether children with obesity solved mental hand rotation tasks using an egocentric perspective (i.e. motor imagery) and if performance was related to their motor competence. Methods.Fifty children (age range: 7-11 y) of which 19 with obesity and motor impairments (OB-) as assessed by the Movement Assessment Battery for Children ( $2^{\text {nd }}$ version), 13 with obesity without motor impairments $(\mathrm{OB}+)$ and 18 control children with a healthy weight (HW) and normal motor competence, were submitted to a classic hand rotation task. Sitting at a desk the children were instructed to indicate the laterality of a picture of a hand as quickly and as accurately as possible. Results. The findings indicate no differences in response time between groups $(2648 \mathrm{~ms}, 2558 \mathrm{~ms}, 2664 \mathrm{~ms}$ for OB-, OB+ and $\mathrm{HW}$ respectively). The OB- group, however, had significantly lower accuracy rates and inverse efficiency scores than the HW group (Accuracy: 72\% vs. 89\%; Inverse efficiency: 4428 vs. 3238). No difference was observed in accuracy and inverse efficiency between the OB+ and HW group (Accuracy: 86\%; Inverse efficiency: 3432). In all groups, slower and more error-prone responses were observed when the angle of rotation was larger and when the hand on display was incongruent with the posture of the participants, which indicates that judgments where made from an egocentric perspective and involved motor imagery. Conclusion.All children, including those with obesity, appear to engage in motor imagery. This notion needs to be investigated further in children with obesity and motor impairments, given their generally lower accuracy and decreased efficiency, which may 
indicate a reduced mental rotation capacity. 


\section{The association between mental rotation capacity and}

2 motor impairment in children with obesity - an

3 exploratory study

4

5

6 Frederik Joris André Deconinck ${ }^{1}$, Eva D'Hondt ${ }^{2}$, Karen Caeyenberghs ${ }^{3}$, Matthieu Lenoir ${ }^{1}$,

7 Mireille Johanna Cornelia Maria Augustijn ${ }^{1}$

8

$9 \quad{ }^{1}$ Department of Movement and Sports Sciences, Ghent University, Ghent, Belgium

$10{ }^{2}$ Faculty of Physical Education and Physiotherapy, Vrije Universiteit Brussels, Brussels,

11 Belgium

$12{ }^{3}$ Mary MacKillop Institute for Health Research, Australian Catholic University, Melbourne,

13 Australia

14

15 Corresponding Author:

16 Frederik JA Deconinck

17 Watersportlaan 2, 9000 Ghent, Belgium

18

19 Email address: frederik.deconinck@ugent.be 


\section{Abstract}

21 Background. Motor impairments are relatively common in children with obesity and evidence suggests that these difficulties go beyond those expected based on the extra weight. This study aimed to investigate the mental rotation capacity in children with obesity, i.e. the ability to mentally view and rotate spatial information, which is a function of both visual-spatial and action representation processes. More in particular, we examined whether children with obesity solved mental hand rotation tasks using an egocentric perspective (i.e. motor imagery) and if performance was related to their motor competence.

Methods. Fifty children (age range: 7-11 y) of which 19 with obesity and motor impairments (OBwithout motor impairments $(\mathrm{OB}+)$ and 18 control children with a healthy weight $(\mathrm{HW})$ and normal motor competence, were submitted to a classic hand rotation task. Sitting at a desk the children were instructed to indicate the laterality of a picture of a hand as quickly and as accurately as possible.

Results. The findings indicate no differences in response time between groups $(2648 \mathrm{~ms}, 2558 \mathrm{~ms}$, 2664ms for OB-, OB+ and HW respectively). The OB- group, however, had significantly lower accuracy rates and inverse efficiency scores than the HW group (Accuracy: 72\% vs. 89\%; Inverse efficiency: 4428 vs. 3238). No difference was observed in accuracy and inverse efficiency between the OB+ and HW group (Accuracy: 86\%; Inverse efficiency: 3432). In all groups, slower and more error-prone responses were observed when the angle of rotation was larger and when the hand on display was incongruent with the posture of the participants, which indicates that judgments where made from an egocentric perspective and involved motor imagery.

Conclusion. All children, including those with obesity, appear to engage in motor imagery. This generally lower accuracy and decreased efficiency, which may indicate a reduced mental rotation capacity. 


\section{Introduction}

47 While recent figures indicate that the increase in body mass index (BMI) in children and adolescents has plateaued in high-income countries, the level is still at an all-time high. Moreover, trends still are accelerating in other parts of the world (e.g. in Asia), which makes pediatric obesity arguably one of today's largest health challenges (1-3). There is indisputable evidence that children with obesity are more likely to stay obese during adolescence and adulthood, which is associated with an increased risk for non-communicable diseases such as cardiovascular diseases, diabetes, musculoskeletal problems and certain types of cancer (4). The focus of the current paper is on the motor competence of children with obesity, a factor that has received increased attention over the past years (e.g. (5-7). Based on a number of studies, the

There is now compelling evidence that the general motor competence of children with obesity is significantly below the level of their peers. On average children with obesity have lower scores on gross and fine motor skills as well as balance (e.g. (8-11)). This is perhaps not surprising, because excess (fat) mass leads to greater inertial forces, which are harder to control and coordinate and therefore hamper movement. Indeed, we found that weight loss after a multidisciplinary treatment is accompanied with an increase in motor competence and may explain up to around $25 \%$ of the improvement in gross motor skills $(12,13)$. It is important to note, however, that the motor impairment found in children with obesity cannot solely be attributed to the presence of excess (fat) mass. In fact, according to our previous work between 50 and $70 \%$ of children with obesity demonstrate levels of motor competence below the $5^{\text {th }}$ percentile, which would indicate a motor impairment that would be associated with more fundamental motor control problems $(6,7,9)$. For example, even in reaction time and eye-hand coordination tracking tasks children with obesity perform slower and less accurate than healthy weight control children $(8,10,14,15)$. These fine motor tasks require only small movements of arm and hand, which makes the interference of excessive mass negligible. Instead, difficulties during the execution of such tasks suggest deficient central processes related to the perception, planning and control of motor actions similar to those found in children with mild motor impairments, such as developmental coordination disorder (DCD) (16).

Although the body of evidence for these more fundamental motor problems in individuals with obesity is currently limited, the findings are compelling enough to warrant further consideration. Motor problems of this kind may pose an extra threat to the individual's health, given the relationship with physical activity, fitness, and mental disorders (17-19), and therefore require specific attention. In order to explore the notion of fundamental motor problems in this population further, the current study investigates one of the processes underlying action planning and control, i.e. mental rotation. 
86 Mental rotation can be defined as the ability to mentally view a representation of spatial 87 information and to transform this representation through rotation (20). This ability, which 88 involves both visual-spatial and action representation processes, is typically tested by asking a 89 subject to judge whether a 2D or 3D geometric shape on display is identical to a reference shape 90 that may have a different orientation (see Figure 1). Chronometric studies have demonstrated that 91 the response time of this judgment increases with increasing angular disparity between the 92 stimulus on display and the reference (20). Moreover, neuroimaging studies have shown that 93 mental rotation engages motor areas, such as the premotor and supplementary motor area (21), 94 especially in a hand laterality judgement task, where the participant is required to indicate the 95 laterality of a normal of mirror-reversed of a hand (HLT; see Figure (22-25)). In this case it is 96 thought that the subject uses an egocentric ( $1^{\text {st }}$ person) perspective and solves the task by imagining moving his/her own hand into the position of the stimulus. For that reason, this type of

98 99

100

101

102

103

104

105

106

107

108

109

110

111

112

113

114

115

116

117

118

119

120

121

122

123

124

125 mental rotation is referred to as motor imagery. Interestingly, motor imagery is embodied and obeys the same rules as actual movements, which implies that the response times in a motor imagery task such as the HLT, are longer for laterally rotated stimuli than for medially rotated stimuli (25). The explanation is that a lateral hand rotation is more complex than a medial rotation due to the anatomical structure and biomechanical constraints (25-27). Furthermore, response times are longer when the posture of the subject is incongruent with the posture of the stimulus, e.g. participants hold hands with palms down while the hand on display is faced with palms up. Mental rotation tasks may also be solved from a $3^{\text {rd }}$ person or object-based perspective. This type of mental rotation uses a non-embodied approach, meaning that responses do not obey the anatomical and biomechanical constraints that act on the body, while there is a linear relationship between the response times and angular disparity between the stimuli $(20,26,27)$.

The ability to mentally manipulate body or object-related information develops through childhood. Although there seems to be considerable interindividual variation, the increase in performance may start from 3 years onwards and is a function of the degree of familiarity with the stimulus rotation, processing time and spatial memory (28-30). Given the link with spatial memory, it is evident that mental rotation ability is related to problem solving (31) and the acquisition of mathematical knowledge (32). The special case of solving mental rotation tasks using an egocentric perspective involving motor imagery increases through childhood, between the ages of 5 and 12 and as a function of an individual's internal action representation capacity $(26,33,34)$. Moreover, accumulating evidence indicates that motor imagery is related to actual motor competence. For example, Kaltner and colleagues have recently demonstrated that very active individuals ( 5 training sessions per week on average) outperform their less active peers ( 1 training session per week on average) on a task evoking egocentric mental rotation (35). Other research shows that individuals with DCD, a neurodevelopmental disorder characterized by difficulties with the acquisition and execution of motor skills, consistently show slower and/or decreased accuracy on the hand laterality task (36-42). Some researchers have also found

Peer) reviewing PDF | (2019:05:38022:3:0:NEW 22 Oct 2019) 
126

127

128

129

130

131

132

133

134

135

136

137

138

139

140

141

142

143

144

145

146

147

148

149

150

151

152

153

154

155

156

157

158

159

160

161

162

163

164

165

atypical response profiles that reflect the use of an $3^{\text {rd }}$ person perspective (43). In these cases, the laterality of the hand would be judged by mentally rotating the hand on display in relation to the surroundings as if it was an object, using other cues (e.g. side of the thumb) than those in motor imagery. The finding that the performance in the hand laterality task is impaired in individuals with DCD, is suggested to indicate compromised internal representations in this population, which would explain difficulties with predictive control $(44,45)$.

Jansen et al. found that the mental rotation capacity of 10-year-old children who are overweight was impaired in a typical letter rotation task (46). In this task two letters are presented alongside each other and the participant is instructed to indicated whether the letter on the right is the same or a mirror-reversed image of the one on the left. While the response time and the performance profile were similar to that of their healthy weight counterparts, children with overweight had significantly larger error rates, especially in the more complex trials (i.e. when the angular disparity was larger). Given that only non-body related stimuli (i.e. letters) were used, the findings of this mental rotation task suggest compromised visual-spatial function, consistent with previous observations in this population $(47,48)$. Interestingly, though, a regression analysis indicated that almost $30 \%$ of the variance in mental rotation performance was explained by children's motor competence. In a second study on mental rotation in individuals with obesity, this time in adolescents, Kaltner et al. contrasted stimuli that elicited either an egocentric or object-based strategy (49). The adolescents with obesity were found to be slower and less accurate than their counterparts with a healthy weight in both conditions. There appeared to be no indication that egocentric or object-based rotation would be more impaired, however, the finding that individuals with obesity were affected more by the angular disparity seemed to suggest a more generalized difficulty with mental rotation. The design of this study was relatively simple and did not check the influence of hand posture or direction of rotation of the stimuli, which are known manipulations to test whether the response involves an embodied perspective, and thus, an internal action representation (27).

The current study set out to investigate the notion of impaired mental rotation in children with obesity further. More specifically, the aim was to investigate the role of motor processes in mental rotation in children with obesity by comparing their performance with typically developing peers and be checking the influence of anatomical and biomechanical constraints on motor imagery. Based on previous research, slower, less accurate performance, or reduced influence of anatomical or biomechanical constraints were considered to indicate impaired motor imagery ability. A second aim was to explore to what extent potential mental rotation difficulties in children with obesity were related with the reduced motor competence found in this population. To this end, the performance on the mental rotation task of children with obesity and concurrent motor impairments was contrasted with that of children with obesity without clear cut motor impairments. 


\section{Materials \& Methods}

168 Participants

169 For this study we initially recruited fifty-seven children, aged between 7 and 11 years. Thirty-

170 two participants were children with obesity ( 14 boys, 18 girls; mean age $=9.6 \pm 1.1$ ), recruited

171 from a specialized rehabilitation center at the start of the actual treatment. Obesity was

172 determined using the international cut-off points standardized for age published by Cole and

173 Lobstein (50). All but five children of this group (2 boys, 3 girls) attended a school for special

174 education. These children were free from severe neurological conditions, but required

175 individualized education due to a delay at school. The healthy weight control children $(\mathrm{N}=25$,

17618 boys, 7 girls, mean age $=9.5 \pm 1.6$ ) were randomly selected from a local database of regular

177 primary school children considering the age range of the group with obesity (range: $+/-6$

178 months). The parents of all children gave written informed consent prior to data collection and

179 the protocol of the study was approved by the Ethics Committee of the Faculty of Medicine and

180 Health Sciences of Ghent University (2014/0003).

181

182

To identify children with a motor impairment, we used the Movement Assessment Battery for Children, $2^{\text {nd }}$ version (MABC-2) (51,52). The MABC-2 consists of 8 items, clustered into three domains (i.e. manual dexterity, ball skills and balance) and has good reliability and validity (53). Using the available norms for Dutch children, a standard score and a percentile score, both for the total general motor competence score and per cluster, were calculated for each child. The general motor competence scores indicated that 19 out of 32 children with obesity had a general motor impairment, as indicated by a motor competence score at or below the $5^{\text {th }}$ percentile. This group is labeled OB-. Of the other children with obesity $(\mathrm{OB}+; \mathrm{N}=13)$, six scored above the $16^{\text {th }}$ percentile and seven had scores between percentile 6 and 16, which would indicate being at risk of a motor impairment. Of note, the five children attending a school for special education were all part of the OB- group. In the group with children with HW, 2 children had a general motor competence score at or below the $5^{\text {th }}$ percentile, and 5 scored at or below the $16^{\text {th }}$ percentile. As we wanted to compare the performance of the children with OB against a group without motor impairments we excluded these children from the study, which resulted in sample of 18 children with HW (see Table 1 for an overview of the sample and the division into three groups).

199 Children's body height $(0.1 \mathrm{~cm})$ was measured barefoot using a calibrated stadiometer

200 (Harpenden, Holtain Ltd., Crymych, UK). Additionally, body mass $(0.1 \mathrm{~kg})$ and percentage body

201 fat $(0.1 \%)$ were obtained by means of a digital balance scale with bioelectrical impedance

202 (Tanita, BC420SMA, Weda B.V., Naarden, Holland). BMI ( $\left.\mathrm{kg} / \mathrm{m}^{2}\right)$ was calculated based on

203 body height and body mass. Finally, waist circumference $(0.1 \mathrm{~cm})$ was measured using a flexible 204 tape measure. 
206 The participant's motor competence was measured using the Movement Assessment Battery for 207 Children, $2^{\text {nd }}$ version (MABC-2) (51,52). The MABC-2 consists of 8 items, clustered into three 208 domains (i.e. manual dexterity, ball skills and balance) and has good reliability and validity (53). 209 Using the available norms for Dutch children, the raw scores were converted into standard scores 210 and a percentile score, both for the total general motor competence score and per cluster. In 211 accordance with the $\mathrm{M}-\mathrm{ABC} 2$ guidelines, general motor competence scores at or below the $5^{\text {th }}$ 212 percentile were considered to indicate a motor impairment, scores at or below the $16^{\text {th }}$ percentile 213 were considered to indicate children "at risk" of a motor impairment.

214

215 Motor imagery was tested with a classic hand laterality judgment task (HLT(25)). Single-hand 216 stimuli ( 9 by $8 \mathrm{~cm}$ ) were presented on a laptop screen (Dell Precision M6700, 17-inch) using 217 OpenSesame (version 3.0.7) (54). The participant sat at a distance of approximately $60 \mathrm{~cm}$ from 218 the screen and was instructed to indicate the laterality of the stimulus by pressing the keyboard 219 (i.e. letter "d" or "k" on a qwerty keyboard for a left or right hand, respectively), while imagining 220 that the hand on display was his/her own hand. The pictures of the hands were presented with 221

222

223

224

225

226

227

228

229

230

231

232

233

234

235

236

237

238

239

240

241

242

243

244

245 palm facing up or down at an angle of $0^{\circ}, 60^{\circ}, 120^{\circ}, 180^{\circ}, 240^{\circ}$, or $300^{\circ}$ (see Figure 2). Before presentation of the stimulus a fixation cross was shown in the center of the screen, which was replaced by the actual hand stimulus after a random interval between 1300 and $1800 \mathrm{~ms}$ to avoid anticipatory responses. After a practice and familiarization period of five trials, during which it was ensured that the participants understood the instructions, three blocks of trials were recorded. Each block contained two trials per combination of stimuli [ 2 hands (left, right), 2 sides (palm, back), 6 orientations $\left(0^{\circ}-300^{\circ}\right) ; \mathrm{N}=24$ ], giving a total of 144 trials per participant. Per trial, the software recorded the accuracy (correct or incorrect) and the response time (RsT) to the nearest ms.

\section{Analysis and statistics}

After deletion of anticipatory responses (RsT $<250 \mathrm{~ms}$ ) and late or absent responses (RsT $\geq$ $8000 \mathrm{~ms}$ ), mean RsTs of the remaining trials (correct and incorrect) were computed at each of the stimulus presentation conditions per individual. Note that the orientations of both hands were flipped such that angles between $0^{\circ}$ and $180^{\circ}$ represented medial rotations; angles between $180^{\circ}$ and $360^{\circ}$ represented lateral rotations. In addition to that, accuracy (ACC) was calculated as the proportion of correct responses at each of the stimulus presentation conditions per individual. As preliminary analysis indicated that there was a positive linear relationship between overall mean RsT and ACC, it was deemed appropriate to calculate the inverse efficiency score (IES), by dividing the RsT by the proportion of correct responses at each stimulus presentation (Townsend \& Ashby, 1978; Townsend \& Ashby, 1983). IES combines speed and error in one metric, yet it inflates variance disproportionally in cases were proportion correct is below chance (see Bruyer $\&$ Brysbaert (57) for a detailed argumentation). The proportion correct in our data ranged from 0.48 to 0.97 , therefore it was decided to calculate IES only for those subjects who had proportion correct scores above chance level. Based on a binomial distribution with $\mathrm{p}=0.50$ for each trial, 
246 individual performance was considered significantly above chance level when more than 76 out 247144 trials $(52.8 \%)$ were correct.

248

249

250

251

252

253

254

255

256

257

258

259

260

261

262

263

264

265

266

267

268

269

270

271

272

273

274

275

276

277

278

279

280

281

282

283

284

285

Between-group differences in anthropometric measurements and $\mathrm{M}-\mathrm{ABC} 2$ scores were examined using separate univariate ANOVAs. To investigate group differences in motor imagery performance three separate repeated measures ANOVA were run for the dependent variables RsT, ACC and IES with Group (OB-, OB+, HW) as between groups factor and Hand (left, right), Side (palm, back), and Angle $\left(0^{\circ}, 60^{\circ}, 120^{\circ}, 180^{\circ}, 240^{\circ}, 300^{\circ}\right)$ as within group factors. Within these analyses our first focus was on the effect of rotation, including potential interactions with the factor Group. Larger RsTs and smaller ACC for greater deviations from the normal orientation (i.e. with the fingers pointing upwards) were indicative of the use of mental rotation to judge laterality. Secondly, we looked at the difference between medial and lateral rotations and the effect of Side to examine whether the anatomical and biomechanical constraints that affect actual movements also hold for mental rotations. Here, smaller RsTs for medial vs. lateral rotations and for hand back vs. hand palm were indicating the use of motor imagery. All analyses were run with IBM SPSS Statistics version 25. Effects with $p<0.05$ were considered significant and partial eta squared values $\left(\eta^{2}\right)$ were reported to indicate effect size where appropriate. To correct for multiple comparisons, Bonferroni adjustments were applied.

\section{Results}

\section{Individual characteristics}

Descriptive statistics of the anthropometric measurements and motor competence scores are shown in Table 1 . The ANOVAs revealed that children with obesity (OB- and $\mathrm{OB}+$ ) were significantly heavier $(\mathrm{p} \leq 0.001)$ and had a higher percentage of body fat $(\mathrm{p} \leq 0.001)$, waist circumference $(p \leq 0.001)$ and body mass index $(p \leq 0.001)$ compared to HW controls. No significant differences in anthropometric measurements were observed between the $\mathrm{OB}+$ and OB- group ( $>0.05$ ). For general motor competence, significant between-group differences were observed, with the HW group performing better than the $\mathrm{OB}+(\mathrm{p}=0.016)$ and $\mathrm{OB}$ - group $(p<0.001)$, and the OB+ group performing better than the OB- group $(p<0.001)$. A similar between-group difference was found for the sub-score on balance $(\mathrm{HW}>\mathrm{OB}+, \mathrm{p}=0.007$; $\mathrm{HW}>\mathrm{OB}-, \mathrm{p}<0.001 ; \mathrm{OB}+>\mathrm{OB}-, \mathrm{p}<0.001)$. For manual dexterity and ball skills the performances of the $\mathrm{HW}$ and $\mathrm{OB}+$ group did not differ, but both groups had significantly higher scores than the OB-group (manual dexterity: $\mathrm{HW}>\mathrm{OB}-, \mathrm{p}<0.001$; $\mathrm{OB}+>\mathrm{OB}-, \mathrm{p}=0.002$; ball skills: $\mathrm{HW}>\mathrm{OB}-$, $\mathrm{p}=0.001 ; \mathrm{OB}+>\mathrm{OB}-, \mathrm{p}=0.016)$.

\section{Mental rotation and motor imagery performance}

The ANOVAs indicated that the effect of Hand stimulus (right or left) was not significant and was not involved in any of the interaction effects for any of the dependent variables (RsT, ACC, and IES). Therefore, this independent factor was removed from further analyses. 
287 For the RsT, we observed a significant effect of Side $\left[F(1,47)=41.689, p<0.001, \eta^{2}=0.470\right]$, 288 Angle $\left[\mathrm{F}(5,235)=35.225, \mathrm{p}<0.001, \eta^{2}=0.428\right]$, and an interaction effect between these factors 289 [Side $x$ Angle $F(5,235)=18.237, p<0.001, \eta^{2}=0.280$ ]. Further investigation indicated that 290 responses to stimuli of hand palms were generally slower than responses to those showing the 291 back of the hand ( $2910 \pm 98 \mathrm{~ms}$ vs. $2543 \pm 88 \mathrm{~ms})$. For the effect of Angle, it was found that for 292 both sides RsTs to medially rotated stimuli $\left(60^{\circ}\right.$ and $\left.120^{\circ}\right)$ were smaller than RsTs to laterally 293 rotated stimuli $\left(240^{\circ}\right.$ and $\left.300^{\circ}\right)$, however, this effect was more pronounced for hand back than 294 for palm of hand (see Figure 3A and 3B). A main effect of Group or any interaction with this 295 factor remained absent.

296

Proportion correct (ACC) was smaller in OB- group $(72.0 \pm 2.0 \%)$ compared with the $\mathrm{OB}+(85.7$

298

299

300

301

302

303

304

305

306

307

308

309

310

311

312

313

314

315

316

317

318

319

320

321

322

323

324

325 $\pm 3.6 \%$ ) and HW group [88.5 $\pm 3.0 \%$; main effect of Group: $\left.F(2,47)=7.525, p=0.001, \eta^{2}=0.243\right]$. No difference was found in ACC between the HW and $\mathrm{OB}+$ children. Furthermore, a main effect of Side $\left[F(1,47)=19.623, p<0.001, \eta^{2}=0.295\right]$ and Angle $\left[F(5,235)=15.224, p<0.001, \eta^{2}=0.245\right]$, as well as an interaction between these two factors was observed $[F(5,235)=5.823, p<0.001$, $\left.\eta^{2}=0.110\right]$. Closer inspection of these effects revealed better ACC when the stimulus was rotated over $0^{\circ}, 60^{\circ}$ or $120^{\circ}$ vs. rotations over $180^{\circ}$ or $240^{\circ}$, with different profiles for palms and backs (see Figure 3C and 3D). No interactions with Group were found.

Five participants, one of the HW and four of the OB- group, demonstrated ACC scores below chance (range: $47.9-52.1 \%$ ). After removing the results of these participants, the ANOVA on the IES indicated a main effect of the factors Group $\left[F(2,40)=3.384, p=0.044, \eta^{2}=0.145\right]$, Side $\left[F(1,40)=13.410, p=0.001, \eta^{2}=0.251\right]$ and Angle $\left[F(5,200)=16.266, p<0.001, \eta^{2}=0.289\right]$. Furthermore, there was a two-way interaction between Side and Angle $[F(5,200)=3.894$, $\left.\mathrm{p}=0.002, \eta^{2}=0.089\right]$ and a three-way interaction between Group, Angle and Side $\left[F(10,200)=2.253, p=0.016, \eta^{2}=0.089\right]$. Post-hoc inspection showed that efficiency was generally better (i.e. IES smaller) for back of the hand vs. hand palm. In addition, the HW group had better IES than the OB- group ( $\mathrm{p}=0.050)$, in particular at angles of $0^{\circ}, 240^{\circ}$ and $300^{\circ}$ for palms and $180^{\circ}, 240^{\circ}$ and $300^{\circ}$ for backs. No significant difference was found between the OB- and the $\mathrm{OB}+$ group, or between the $\mathrm{OB}+$ group and the HW group (see Figure $3 \mathrm{E} \& 3 \mathrm{~F}$ ).

\section{Discussion}

This study set out to examine the capacity to enlist motor imagery during a mental rotation task in children with obesity. In addition to this, it was investigated whether performance on this task was related to the children's motor competence. To this end, the response time, accuracy and efficiency of the children with obesity with scores below the $5^{\text {th }}$ percentile on the MABC-2 (OB) was compared to those of the other children with obesity ( $\mathrm{OB}+$ ) and those with healthy weight (HW) using a classic hand laterality task. It was found that responses of children with obesity 
326 (OB- and $\mathrm{OB}+$ ) were as fast as those in healthy weight control children (HW). Proportion of 327 correct responses and efficiency were smaller in the group with obesity and motor difficulties 328 (OB-), but the judgements were influenced by the side of the stimulus and its angle of rotation to 329 a similar extent in all three groups.

330

331

332

333

334

335

336

337

338

339

340

341

342

343

344

345

346

347

348

349

350

351

352

353

354

355

356

357

358

359

360

361

362

363

364

365

Consistent with previous studies, stimuli with greater angular deviations resulted in slower responses, which indicates that mental rotation was used to judge the laterality of the hand on display. Indeed, the hand laterality task requires visual spatial cognition and reasoning, and it has been shown that the duration of these processes increases with angular disparity between the orientation of the stimulus and the "normal" orientation. There is evidence that this ability is related to problem solving (31) and the acquisition of mathematical knowledge (32), and is likely to be involved in sport and movement skills $(35,58)$. In keeping with this, the children with obesity and motor difficulties in the present study made more errors and were less efficient than controls. Proportion correct and inverse efficiency scores of children with obesity without motor problems were not different from those in healthy weight children. This difference between the two groups with obesity suggests reduced spatial reasoning skills, but only in those with severe motor difficulties.

Another objective of this study was to investigate the embodied nature of mental rotation or motor imagery in hand laterality judgments in children with obesity with and without motor impairments. This is important as the ability to enlist motor imagery is linked to a person's internal action representation capacity, which is deemed to be essential for motor planning and control (45). In all three groups response times were longer when the side of the hand on display was incongruent with the posture of the participant (longer response time for palm vs. back). In addition, stimuli rotated to the lateral side $\left(300^{\circ}\right.$ and $\left.240^{\circ}\right)$ led to longer response times than stimuli rotated to the medial side $60^{\circ}$ and $120^{\circ}$. In other words, the behavior of all three groups conformed to the anatomical and/or biomechanical constraints that act on actual hand rotations. This indicates that, irrespective of group, hand laterality judgements appear to be solved automatically using embodied mental spatial transformations of the viewer ( $1^{\text {st }}$ person perspective) rather than of the viewed object ( $3^{\text {rd }}$ person perspective). This is in contrast to what has been observed in other populations with motor impairments, where motor imagery is only used after specific instruction $(59,60)$.

Our results corroborate earlier findings of Kaltner et al. (47), who found that adolescents with obesity were able to engage in egocentric transformations, yet with slower response times than their healthy weight peers for larger angular deviations. Despite the fact that the responses in this mental rotation task appear to be embodied, and involve motor imagery, the overall performance of children and adolescents with obesity is reduced, yet in the current study only in those with motor impairments. Preliminary evidence suggests that the poor performance in this task is associated with reduced excitability of the primary motor cortex when engaging in motor

Peer) reviewing PDF | (2019:05:38022:3:0:NEW 22 Oct 2019) 
366

367

368

369

370

371

372

373

374

375

376

377

378

379

380

381

382

383

384

385

386

387

388

389

390

391

392

393

394

395

396

397

398

399

400

401

402

403

404

405

imagery (61). It thus seems that the visual-spatial and action representation processes underlying motor imagery are affected in this group. Given the known relationship between impaired motor imagery and motor control issues (40), our findings provide support to the notion that the motor impairment of these children is not just a matter of excessive weight but may be related to more fundamental deficits in motor control. Indeed, previous research signaled the potential relationship between DCD and obesity $(62,63)$, calling for special attention in the treatment of this group.

Clearly, the relationship between obesity and motor control problems is complex, and the present study does not allow to infer cause and effect. The association found between obesity, motor impairments and mental rotation deficits warrants further investigation. In this respect, it is also relevant to note that children with motor impairments often withdraw from movement opportunities (64). Limited movement activity may, in turn, have a negative impact on the development of spatial cognition, so the relationship between motor competence and mental rotation capacity is likely to be reciprocal (65). Furthermore, nutritional research in rats has shown that diet-induced obesity due to excess sucrose intake may lead to impaired spatial learning and long-term spatial memory (66). These findings highlight that obesity is a multifaceted problem that requires a multidisciplinary approach with attention for dietary and lifestyle factors, as well as cognitive and motor competence $(67,68)$.

By contrasting the mental rotation performance of children with obesity with and without motor impairments, the current study has provided an extra dimension to earlier findings of Jansen and colleagues $(46,49)$. Here, we have shown that deficits in mental rotation are a particular problem when obesity is accompanied by motor impairments, however, a number of limitations need to be considered. First, the design lacks a group of HW children with motor impairments. This would have enabled us to directly assess the variance related to the relevant factors body weight/obesity and motor impairment and the interaction between the two. Secondly, the children with obesity were recruited from a specialized rehabilitation center, meaning that sampling was not fully randomized. In fact, the children are referred to this center by their general practitioner or pediatrician based on the severity of their weight problem and failure of conventional care. Our findings may therefore pertain to the more severe cases of obesity. Furthermore, data on the intellectual capacity or processing speed of our sample were not available. Given that spatial cognition is known to correlate with mathematical capacity it would have been desirable to control for this factor. In this respect, it is also important to note that five out of 19 children of the OB- group attended a school for special education. While these children were free from neurological conditions, (subtle) neuropsychological issues cannot be excluded. As for processing speed, we know that children with obesity demonstrate increased reaction times in a simple reaction time task (15). Whether or not the current findings reflect a specific issue with spatial processing and action representation or rather a general slowness, should be the subject of future research. Finally, our results only provide insight into one specific aspect of the

PeerJ reviewing PDF | (2019:05:38022:3:0:NEW 22 Oct 2019) 
406 central processes related to spatial cognition and motor control, i.e. mental rotation capacity and 407 motor imagery. To unravel the motor impairments of children with obesity further, more 408 research is needed.

409

410

411

412

413

414

415

416

417

418

419

420

421

422

423

424

425

426

427

428

429

430

431

432

433

434

435

436

437

438

439

440

441

442

443

444

445

446

447

\section{Conclusions}

The results of this study demonstrate that children with obesity engage in motor imagery during hand laterality judgments in this mental rotation tasks, regardless of the fact that they have motor impairments. The performance of children with obesity and motor impairments is, however, flawed (i.e. slower and less efficient) compared to their counterparts without motor impairments and children with a healthy weight. It thus seems that the visual-spatial and action representation processes underlying motor imagery are affected in this group. A deficit in these processes lends support to the notion that the motor impairments are related to more fundamental deficits in motor control and are not just a matter of excessive weight. Due to the specific nature of the sample and the lack of potential contributing factors, e.g. intellectual capacity, our findings should be treated with caution, however, for practitioners it is important to acknowledge the potential presence of motor impairments in children with obesity.

\section{Acknowledgements}

The authors are very grateful to all participants and their parents, the staff from the rehabilitation center "Zeepreventorium" (De Haan, Belgium) and the board of the participating schools. All authors conceived and designed the experiment.

\section{References}

1. Abarca-Gómez L, Abdeen ZA, Hamid ZA, Abu-Rmeileh NM, Acosta-Cazares B, Acuin C, et al. Worldwide trends in body-mass index, underweight, overweight, and obesity from 1975 to 2016: a pooled analysis of 2416 population-based measurement studies in 128.9 million children, adolescents, and adults. Lancet. 2017;390(10113):2627-42.

2. Ng M, Fleming T, Robinson M, Thomson B, Graetz N, Margono C, et al. Global, regional, and national prevalence of overweight and obesity in children and adults during 1980-2013: A systematic analysis for the Global Burden of Disease Study 2013. Lancet. 2014;384(9945):76681 .

3. Lobstein T, Jackson-Leach R, Moodie ML, Hall KD, Gortmaker SL, Swinburn BA, et al. Child and adolescent obesity: Part of a bigger picture. Lancet [Internet]. 2015;385(9986):2510-20. Available from: http://dx.doi.org/10.1016/S0140-6736(14)61746-3

4. Simmonds M, Llewellyn A, Owen CG, Woolacott N. Predicting adult obesity from childhood obesity: A systematic review and meta-analysis. Obes Rev. 2016;17(2):95-107. 
448 5. Robinson LE, Stodden DF, Barnett LM, Lopes VP, Logan SW, Rodrigues LP, et al. Motor

449

450

451

452

453

454

455

456

457

458

459

460

461

462

463

464

465

466

467

468

469

470

471

472

473

474

475

476

477

478

479

480

481

482

483

484

485

486

487

488

489

490

491

492

493

494

495

496

497

498 competence and its effect on positive developmental trajectories of health. Sport Med. 2015;45(9):1273-84.

6. D'hondt E, Deforche B, De Bourdeaudhuij I, Lenoir M. Relationship Between Motor Skill and Body Mass Index in 5-to 10-Year-Old Children. Adapt Phys Act Q. 2009;26:21-37.

7. Augustijn MJCM, Deconinck FJA, D'Hondt E, van Acker L, de Guchtenaere A, Lenoir M, et al. Reduced motor competence in children with obesity is associated with structural differences in the cerebellar peduncles. Brain Imaging Behav. 2018;12(4):1000-10.

8. Gentier I, D'Hondt E, Shultz S, Deforche B, Augustijn M, Hoorne S, et al. Fine and gross motor skills differ between healthy-weight and obese children. Res Dev Disabil. 2013;34(11):4043-51.

9. D'Hondt E, Deforche B, Vaeyens R, Vandorpe B, Vandendriessche J, Pion J, et al. Gross motor coordination in relation to weight status and age in 5- to 12-year-old boys and girls: A crosssectional study. Int J Pediatr Obes. 2011;6(2-2).

10. Petrolini N, Iughetti L, Bernasconi S. Difficulty in visual motor coordination as a possible cause of sedentary behaviour in obese children. Vol. 19, International journal of obesity and related metabolic disorders. 1995. p. 928.

11. Deforche BI, Hills AP, Worringham CJ, Davies PSW, Murphy AJ, Bouckaert JJ, et al. Balance and postural skills in normal-weight and overweight prepubertal boys. Int J Pediatr Obes. 2009;4:175-82.

12. D'Hondt E, Gentier I, Deforche B, Tanghe A, De Bourdeaudhuij I, Lenoir M. Weight Loss and Improved Gross Motor Co-Ordination in Children as a Result of Multidisciplinary Residential Obesity Treatment. Obesity (Silver Spring). 2011;19(10):1999-2005.

13. Augustijn MJCM, D'Hondt E, Leemans A, Van Acker L, De Guchtenaere A, Lenoir M, et al. Weight loss, behavioral change, and structural neuroplasticity in children with obesity through a multidisciplinary treatment program. Hum Brain Mapp [Internet]. 2018 Sep; Available from: http://doi.wiley.com/10.1002/hbm.24360

14. D'Hondt E, Deforche B, De Bourdeaudhuij I, Lenoir M. Childhood obesity affects fine motor skill performance under different postural constraints. Neurosci Lett [Internet]. 2008 Jul;440(1):72-5. Available from: http://www.ncbi.nlm.nih.gov/pubmed/18541379

15. Gentier I, Augustijn M, Deforche B, Tanghe A, De Bourdeaudhuij I, Lenoir M, et al. A comparative study of performance in simple and choice reaction time tasks between obese and healthy-weight children. Res Dev Disabil. 2013;34(9).

16. Wilson PH, Ruddock S, Smits-Engelsman B, Polatajko H, Blank R. Understanding performance deficits in developmental coordination disorder: a meta-analysis of recent research. Dev Med Child Neurol [Internet]. 2013 Mar;55(3):217-28. Available from:

http://www.ncbi.nlm.nih.gov/pubmed/23106668

17. Faught BE, Hay JA, Cairney J, Flouris A. Increased risk for coronary vascular disease in children with developmental coordination disorder. J Adolesc Heal [Internet]. 2005;37(5):376-80.

Available from: isi:000232765500006

18. Cairney J, Dudley D, Kwan M, Bulten R, Kriellaars D. Physical Literacy, Physical Activity and Health : Toward an Evidence - Informed Conceptual Model. Sport Med [Internet]. 2019;49(3):371-83. Available from: https://doi.org/10.1007/s40279-019-01063-3

19. Gagnon-Roy M, Jasmin E, Camden C. Social participation of teenagers and young adults with developmental co-ordination disorder and strategies that could help them: results from a scoping review. Child care Heal Dev. 2016;42(6):840-51.

20. Shephard RN, Metzler J. Mental rotation of three-dimensional objects. Science (80- ). 1971;171(3972):701-3.

21. Richter W, Somorjai R, Summers R, Jarmasz M, Menon RS, Gati JS, et al. Motor Area Activity During Mental Rotation Studied by Time-Resolved Single-Trial fMRI. J Cogn Neurosci [Internet]. 2000;12(2):310-20. Available from: http://www.mitpressjournals.org/doi/10.1162/089892900562129 
499

500

501

502

503

504

505

506

507

508

509

510

511

512

513

514

515

516

517

518

519

520

521

522

523

524

525

526

527

528

529

530

531

532

533

534

535

536

537

538

539

540

541

542

543

544

545

546

547

548

549

22. Kosslyn SM, DiGirolamo GJ, Thompson WL, Alpert NM. Mental rotation of object versus hands: neural mechanisms revealed by positron emission tomography. Psychophysiology. 1998;35:15161.

23. Kosslyn SM, Thompson WL, Wraga M, Alpert NM. Imagining rotation by endogenous versus exogenous forces: Distinct neural mechanisms. Neuroreport. 2001;12(11):2519-25.

24. Parsons LM, Fox PT, Downs JH, Glass T, Hirsch TB, Martin CC, et al. Use of implicit motor imagery for visual shape discrimination as revealed by PET. Nature [Internet]. 1995;375(6526):54-8. Available from: http://www.nature.com/doifinder/10.1038/375054a0

25. Parsons LM. Imagined Spatial Transformations and Feet of One's Hands. 1987;241:178-241.

26. Funk M, Brugger P, Wilkening F. Motor processes in children's imagery: the case of mental rotation of hands. Dev Sci [Internet]. 2005 Sep;8(5):402-8. Available from:

http://www.ncbi.nlm.nih.gov/pubmed/16048512

27. de Lange FP, Helmich RC, Toni I. Posture influences motor imagery: An fMRI study. Neuroimage. 2006;33(2):609-17.

28. Frick A, Hansen MA, Newcombe NS. Development of mental rotation in 3- to 5-year-old children. Cogn Dev [Internet]. 2013;28(4):386-99. Available from:

http://dx.doi.org/10.1016/j.cogdev.2013.06.002

29. Kail R. Processing Time, Imagery, and Spatial Memory. J Exp Child Psychol. 1997;64:67-78.

30. Kail R, Pelligrino J, Carter P. Developmental Changes in Mental Rotation. J Exp Child Psychol. 1980;29:102-16.

31. Geary DC, Saults SJ, Liu F, Hoard MK. Sex Differences in Spatial Cognition, Computational Fluency, and Arithmetical Reasoning. J Exp Child Psychol. 2000;77(4):337-53.

32. Hegarty M, Kozhevnikov M. Types of visual-spatial representations and mathematical problem solving. J Educ Psychol. 1999;91(4):684-9.

33. Caeyenberghs K, Wilson PH, van Roon D, Swinnen SP, Smits-Engelsman BCM. Increasing convergence between imagined and executed movement across development: evidence for the emergence of movement representations. Dev Sci [Internet]. 2009 Apr;12(3):474-83. Available from: http://www.ncbi.nlm.nih.gov/pubmed/19371372

34. Spruijt S, Jongsma MLA, Van Der Kamp J, Steenbergen B. Predictive models to determine imagery strategies employed by children to judge hand laterality. PLoS One [Internet]. 2015;10(5):1-14. Available from: http://dx.doi.org/10.1371/journal.pone.0126568

35. Kaltner S, Riecke BE, Jansen P. Embodied mental rotation : a special link between egocentric transformation and the bodily self. Front Psychol. 2014;5(June):1-11.

36. Adams ILJ, Lust JM, Wilson PH, Steenbergen B. Testing predictive control of movement in children with developmental coordination disorder using converging operations. Br J Psychol [Internet]. 2017;108(1):73-90. Available from: http://doi.wiley.com/10.1111/bjop.12183

37. Adams ILJ, Lust JM, Wilson PH, Steenbergen B. Neuroscience and Biobehavioral Reviews Compromised motor control in children with DCD : A deficit in the internal model ?-A systematic review. Neurosci Biobehav Rev [Internet]. 2014;47:225-44. Available from: http://dx.doi.org/10.1016/j.neubiorev.2014.08.011

38. Deconinck FJA, Spitaels L, Fias W, Lenoir M. Is developmental coordination disorder a motor imagery deficit? J Clin Exp Neuropsychol [Internet]. 2009 Aug;31(6):720-30. Available from: http://www.ncbi.nlm.nih.gov/pubmed/19048429

39. Hyde C, Fuelscher I, Buckthought K, Enticott PG, Gitay MA, Williams J. Motor imagery is less efficient in adults with probable developmental coordination disorder: Evidence from the hand rotation task. Res Dev Disabil [Internet]. 2014;35(11):3062-70. Available from: http://dx.doi.org/10.1016/j.ridd.2014.07.042

40. Fuelscher I, Williams J, Enticott PG, Hyde C. Reduced motor imagery efficiency is associated with online control difficulties in children with probable developmental coordination disorder. Res Dev Disabil [Internet]. 2015;45-46:239-52. Available from:

http://dx.doi.org/10.1016/j.ridd.2015.07.027 
550

551

552

553

554

555

556

557

558

559

560

561

562

563

564

565

566

567

568

569

570

571

572

573

574

575

576

577

578

579

580

581

582

583

584

585

586

587

588

589

590

591

592

593

594

595

596

597

598

599

600

41. Reynolds JE, Licari MK, Elliott C, Lay BS, Williams J. Motor imagery ability and internal representation of movement in children with probable developmental coordination disorder. Hum Mov Sci [Internet]. 2015;44:287-98. Available from: http://dx.doi.org/10.1016/j.humov.2015.09.012

42. Williams J, Thomas PR, Maruff P, Butson M, Wilson PH. Motor, visual and egocentric transformations in children with Developmental Coordination Disorder. Child Care Health Dev [Internet]. 2006 Nov;32(6):633-47. Available from:

http://www.ncbi.nlm.nih.gov/pubmed/17018040

43. Wilson PH, Maruff P, Butson M, Williams J, Lum J, Thomas PR. Internal representation of movement in children with developmental coordination disorder: a mental rotation task. Dev Med Child Neurol [Internet]. 2004;46(11):754-9. Available from: isi:000225066000006

44. Wolpert DM, Diedrichsen J, Flanagan JR. Principles of sensorimotor learning. Nat Rev Neurosci [Internet]. 2011;12(12). Available from: http://dx.doi.org/10.1038/nrn3112

45. Desmurget M, Grafton S. Forward modeling allows feedback control for fast reaching movements. Trends Cogn Sci [Internet]. 2000;4(11):423-31. Available from: wos:000165081400005

46. Jansen P, Schmelter A, Kasten L, Heil M. Impaired mental rotation performance in overweight children. Appetite [Internet]. 2011;56(3):766-9. Available from: http://dx.doi.org/10.1016/j.appet.2011.02.021

47. Li Y, Dai Q, Jackson JC, Zhang J. Overweight is associated with decreased cognitive functioning among school-age children and adolescents. Obesity. 2008;16(8):1809-15.

48. Augustijn MJCM, D’Hondt E, Van Acker L, De Guchtenaere A, Lenoir M, Caeyenberghs K, et al. The role of motor competence and executive functioning in weight loss: a study in children with obesity. J Dev Behav Pediatr. 2018;39(8):642-51.

49. Kaltner S, Schulz A, Jansen P. The association between obesity and mental rotation ability in an adolescent sample. Obes Res Clin Pract. 2017;11(1):127-9.

50. Cole TJ, Lobstein T. Extended international (IOTF) body mass index cut-offs for thinness, overweight and obesity. Pediatr Obes. 2012;7(4):284-94.

51. Henderson SE, Sugden DA, Barnett AL. Movement assessment battery for children - 2: Movement ABC-2: Examiner's manual. London: Psychological Corporation; 2007.

52. Smits-Engelsman BCM, Henderson S, Sugden D, Barnett A. Movement Assessment Battery for Children-2: manual. Amsterdam: Pearson; 2010.

53. Cools W, De Martelaer K, Samaey C, Andries C. Movement skill assessment of typically developing preschool children : A review of seven movement skill assessment tools. J Sport Sci Med. 2009;8:154-68.

54. Mathot S, Schreij D, Theeuwes J. OpenSesame: An open-source, graphical experiment builder for the social sciences. Behav Res Methods. 2012;44(2):314-24.

55. Townsend JT, Ashby FG. Methods of Modeling Capacity in Simple Processing Systems. Cognitive theory (Volume 3). 1978. p. 200-39.

56. Townsend JT, Ashby FG. Stochastic Modeling of Elementary Psychological Processes. Cambridge: Cambridge University Press; 1983.

57. Bruyer R, Brysbaert M. Combining Speed and Accuracy in Cognitive. Psychol Belg. 2011;51:513.

58. Jansen P, Lehmann J, Doren J. Mental rotation performance in male soccer players. 2012;7(10):e48620.

59. Williams J, Thomas PR, Maruff P, Butson M, Wilson PH. Motor, visual and egocentric transformations in children with Developmental Coordination Disorder. Child Care Heal Dev. 2006;32(6):633-47.

60. Williams J, Thomas PR, Maruff P, Wilson PH. The link between motor impairment level and motor imagery ability in children with developmental coordination disorder. Hum Mov Sci [Internet]. 2008;27(2):270-85. Available from: wos:000256575600009

61. Hyde C, Fuelscher I, Williams J, Lum JAG, He J, Barhoun P, et al. Corticospinal excitability 
601 during motor imagery is reduced in young adults with developmental coordination disorder. Res

602 Dev Disabil. 2018;72(May):214-24.

603 62. Cairney J, Hay JA, Faught BE, Hawes R. Developmental coordination disorder and overweight

604

605

606

607

608

609

610

611

612

613

614

615

616

617

618 and obesity in children aged 9-14 y. Int J Obes [Internet]. 2005;29(4):369-72. Available from: isi:000227627300005

63. Hendrix CG, Prins MR, Dekkers H. Developmental coordination disorder and overweight and obesity in children : a systematic review. Obes Rev. 2014;15:408-23.

64. Smyth MM, Anderson HI. Coping with clumsiness in the school playground: Social and physical play in children with coordination impairments. Br J Dev Psychol. 2000;18:389-413.

65. Vasilyeva M, Lourenco SF. Development of spatial cognition. Wiley Interdiscip Rev Cogn Sci. 2012;3(3):349-62.

66. Jurdak N, Lichtenstein AH, Kanarek RB. Diet-induced obesity and spatial cognition in young male rats. Nutr Neurosci. 2008 Apr;11(2):48-54.

67. Liang J, Matheson BE, Kaye WH, Boutelle KN. Neurocognitive correlates of obesity and obesityrelated behaviors in children and adolescents. Int J Obes [Internet]. 2014;38(4):494-506. Available from: http://dx.doi.org/10.1038/ijo.2013.142

68. Simmonds M, Llewellyn A, Owen CG, Woolacott N. Predicting adult obesity from childhood obesity: a systematic review and meta-analysis. 2016;(February):95-107.

619 


\section{Table $\mathbf{1}$ (on next page)}

Descriptive statistics (mean \pm standard deviation) for anthropometric measurements and motor competence of the three groups.

Children with obesity + motor impairment (OB-), children with obesity without motor impairment $(\mathrm{OB}+)$ and children with healthy weight without motor impairment $(\mathrm{HW})$. The final column reports the outcome of the ANOVA to explore between between-group differences. 
1

\begin{tabular}{lcccc}
\hline & OB- & OB+ & HW & ANOVA \\
& N=19 & N=13 & N=18 & F(2,47) \\
\hline Demographic characteristics & & & & \\
Gender (boys / girls) & $6 / 13$ & $7 / 6$ & $14 / 4$ & $/$ \\
Age & $9.9 \pm 1.1$ & $9.2 \pm 1.0$ & $9.5 \pm 1.3$ & 1.269 \\
\hline Anthropometric measurements & & & & \\
\hline Body height (cm) & $145.2 \pm 7.8$ & $141.9 \pm 7.8$ & $140.2 \pm 8.9$ & 1.739 \\
Body weight (kg) & $68.7 \pm 18.3$ & $59.9 \pm 8.8$ & $33.4 \pm 5.7$ & $37.993^{*}$ \\
Body fat (\%) & $44.8 \pm 9.1$ & $43.8 \pm 5.9$ & $16.8 \pm 4.2$ & $93.079^{*}$ \\
Waist circumference (cm) & $94.7 \pm 13.3$ & $91.3 \pm 7.5$ & $61.1 \pm 4.5$ & $67.735^{*}$ \\
Body mass index (kg/m $\left.{ }^{2}\right)$ & $32.2 \pm 6.5$ & $29.7 \pm 2.5$ & $16.9 \pm 1.2$ & $64.891^{*}$ \\
\hline Motor competence & & & & \\
\hline General motor competence & $46.8 \pm 12.2$ & $71.9 \pm 10.1$ & $82.4 \pm 6.5$ & $62.311^{*}$ \\
Manual dexterity & $18.8 \pm 8.1$ & $27.3 \pm 6.2$ & $30.2 \pm 4.3$ & $15.497^{*}$ \\
Ball skills & $14.4 \pm 5.5$ & $19.7 \pm 5.0$ & $21.1 \pm 4.5$ & $8.886^{*}$ \\
Balance skills & $13.6 \pm 5.5$ & $24.9 \pm 7.0$ & $31.1 \pm 3.3$ & $51.394^{*}$ \\
\hline
\end{tabular}

Note. Children with obesity + motor impairment $(\mathrm{OB}-)$, children with obesity without motor impairment $(\mathrm{OB}+)$ and children with healthy weight without motor impairment (HW). The final column reports the outcome of the ANOVA to explore between between-group differences. * $\mathrm{p} \leq 0.001$ 
Figure 1

Four typical stimuli for a mental rotation task.

In $A$ and $B$ the task is to judge whether the $3 D$ shapes on the right is identical to or a mirror reversed image of the reference shape on the left. In $C$ and $D$ the task to judge whether the hand is a left or a right hand.

A
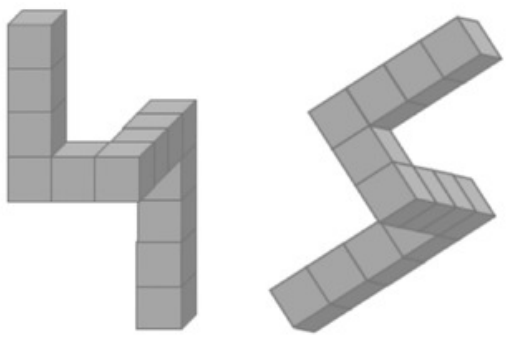

C

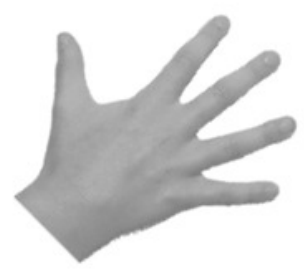

B
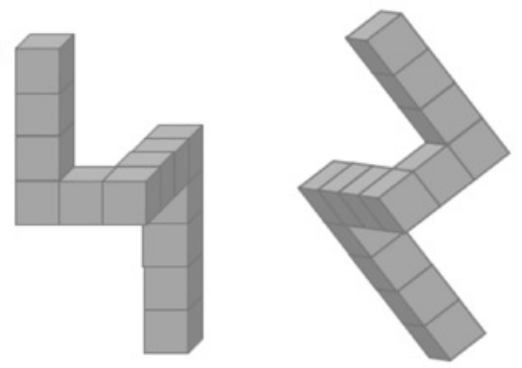

D

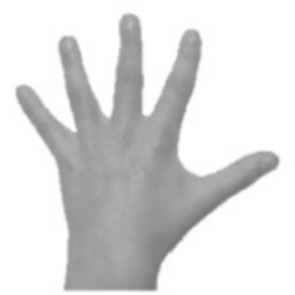




\section{Figure 2}

Illustration of hand stimuli used in the hand laterality judgement task.

The stimuli are shown at each of the six possible angles of rotation. A: Palm of the left hand;

B: Palm of the right hand; C: Back of the left hand; D: Back of the right hand.
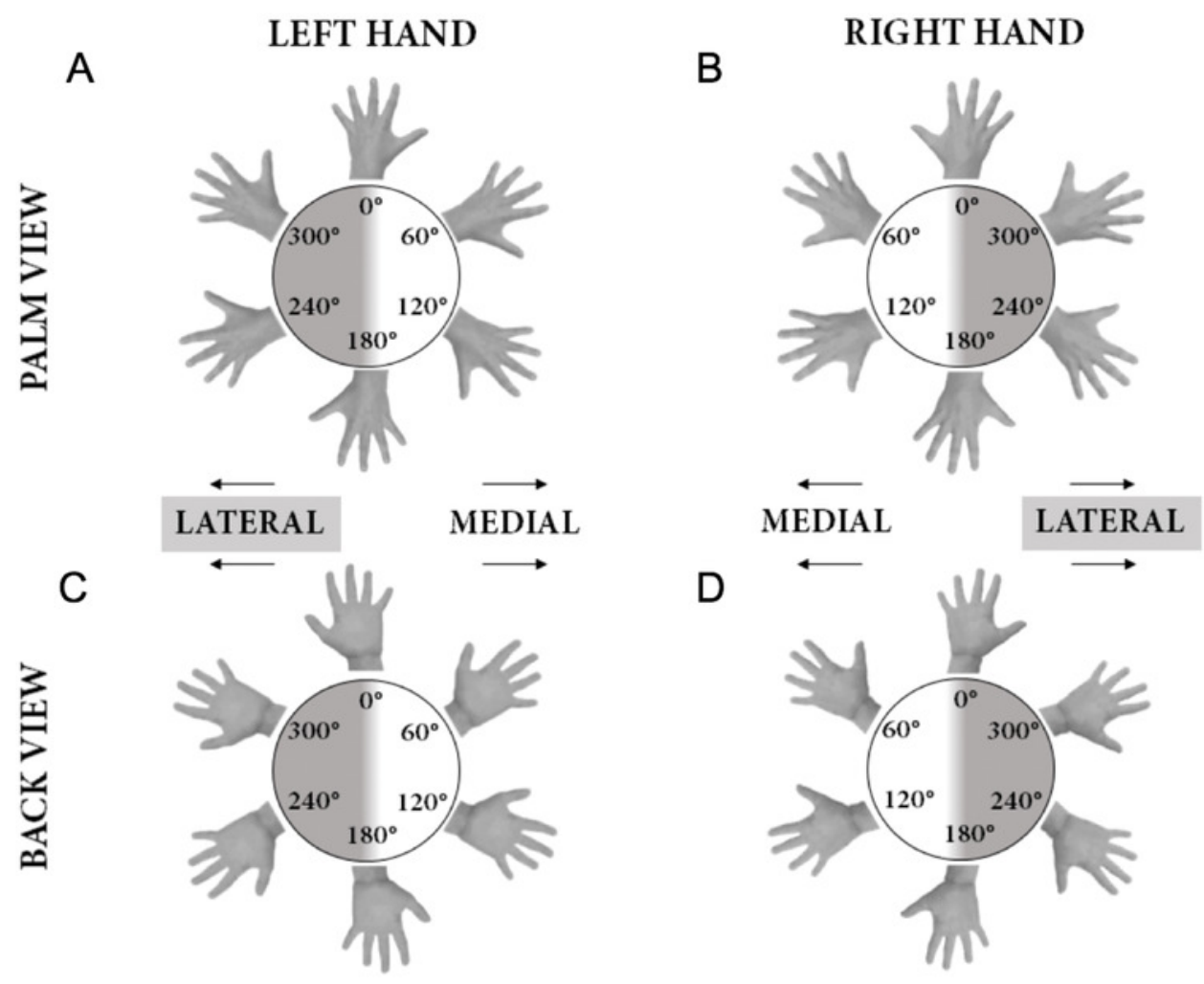


\section{Figure 3}

Response Time $(A \& B)$, Proportion Correct $(C \& D)$ and Inverse Efficiency Score $(E \& F)$.

The group of children with obesity and motor impairments (OB-) is indicated with $\diamond$; Children with obesity without motor impairments $(\mathrm{OB}+)$ are indicated with $\bigcirc$ : Healthy weight control group (HW) is indicated with $\bullet$. The stimulus on display was the palm of the hand in the left panels $(A, C, E)$; the back of the hand is shown in the right panels $(B, D, F)$. 

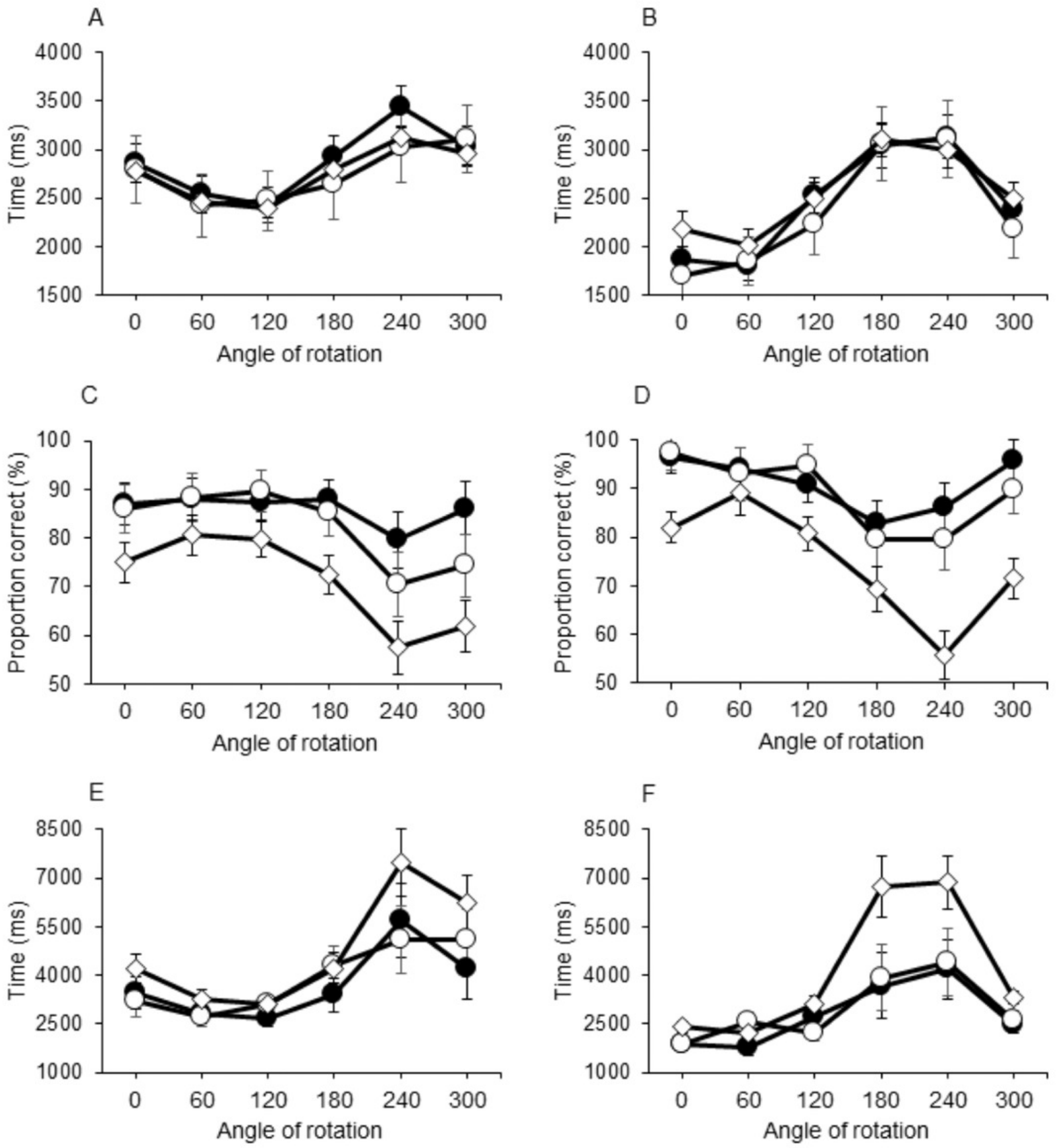